10. Bounhar $y$, Zhang $y$, Goodyer CG, LeBlanc A. Prion protein protects human neurons against Bax-mediated apoptosis. J Biol Chem 2001 . 276: 39145-9.

11. Paitel $\varepsilon$, Fahraeus R, Checler F. Cellular prion protein sensitizes neurons to apoptotic stimuli through Mdm2-regulated and P53-dependent caspase 3-like activation. J Biol Chem 2003; 278: 10061-6.

12. Dupuis L, Mbebi C, Gonzalez de Aguilar JL, et al. Loss of prion protein in a transgenic model of amyotrophic lateral sclerosis. Mol Cell Neurosci 2002; 19: 216-24.
13. Dupuis L, Di Scala F, Gonzalez de Aguilar JL, Rene F, De Tapia M, LoefflerJP. Denervation is not the primary cause of prion protein downregulation occuring in the spinal cord of a transgenic model of amyotrophic lateral sclerosis. Ann Ny Acad Sci 2002; 973: 116-9.

14. Zanusso G, Vattemi G, Ferrari $S$, et al. Increased expression of the normal cellular isoform of prion protein in inclusion-body myositis, inflammatory myopathies and denervation atrophy. Brain Pathol 2001; 11: 182-9.
15. Lampe JB, Walter MC, Reichmann $\mathrm{H}$. Neurodegenerationassociated proteins and inflammation in sporadic inclusion-body myositis. Adv Exp Med Biol 2001; 487: 219-28.

16. Westaway D, DeArmond SJ, Cayetano-Canlas J, et al. Degeneration of skeletal muscle, peripheral nerves, and the central nervous system in transgenic mice overexpressing wild-type prion proteins. Cell 1994; 76: 117-29.
17. Chiesa R, Pestronk A, Schmidt RE, et al. Primary myopathy and accumulation of PrPSc-like molecules in peripheral tissues of transgenic mice expressing a prion protein insertional mutation. Neurobiol Dis 2001; 8: 279-88.

18. Miele $G$, Jeffrey $M$, TurnbullD, Manson J, Clinton, M. Ablation of cellular prion protein expression affects mitochondrial numbers and morphology. Biochem Biophys Res Commun 2002; 291: 372-7.

\title{
NOUVELLE
}

\section{Équations de la soudure épithéliale}

Raphaël Rousset, Luis Almeida, Stéphane Noselli

> La réparation tissulaire, en particulier la fermeture des plaies, est un acte réflexe des tissus permettant d'assurer leur continuité et la défense face aux infections [1]. L'imperfection notoire de la réparation se manifeste par l'apparition de cicatrices d'aspect peu esthétique. De multiples stratégies, le plus souvent empiriques, ont été élaborées au fil du temps pour tendre vers la cicatrisation parfaite (c'est-à-dire rapide et sans trace), mais le remède miracle reste à découvrir. Une condition importante pour l'élaboration de traitements efficaces est le développement de modèles animaux offrant un large éventail d'approches et de méthodologies. L'un de ces modèles est la fermeture dorsale (FD) de l'embryon de drosophile. La FD représente une étape clé de l'embryogenèse au cours de laquelle la région dorsale de l'embryon, occupée par l'amnioséreuse, est recouverte par deux feuillets épithéliaux migrant l'un vers l'autre (Figure 1A) [2, 3]. En l'espace de 2 heures, ils se rejoignent au niveau de la ligne dorsale et fusionnent parfaitement. Vu de dessus, le rapprochement des deux marges de l'ectoderme en cours de migration peut être comparé au clignement d'un œil dont les coins (canthus) se fermeraient avant le centre selon un processus de type «fermeture éclair» (zipping). De façon intéressante, ce processus naturel de soudure épithéliale peut être assimilé à une cicatrisation programmée au cours du développement $[4,5]$.

L'étude génétique, moléculaire et cellulaire de la FD a permis d'identifier trois structures essentielles à ce processus: l'amnioséreuse, l'ectoderme latéral et, délimitant ces deux tissus, la marge de
R. Rousset, S. Noselli : Institut de Recherches Signalisation, Biologie du Développement et Cancer, Cnrs UMR 6543, Université de Nice-Sophia Antipolis,

Centre de Biochimie, Parc

Valrose, 06108 Nice Cedex 2, France.

L. Almeida: Laboratoire J. A. Dieudonné, Cnrs UMR 6621, Parc Valrose, 06108 Nice Cedex 2, France. noselli@unice.fr

l'ectoderme en migration (Figure IA) [2, 3]. Celle-ci est notamment caractérisée par la présence d'un câble riche en actine qui relie chaque cellule [6], ainsi que d'extensions du cytosquelette (filopodes, lamellipodes) importantes pour la suture des marges [7]. Cependant, la contribution relative de ces trois structures au mouvement coordonné de la FD est totalement inconnue. Afin de mieux comprendre les forces et tensions mises 
en jeu, une approche fondée sur l'utilisation de la microchirurgie au laser des trois tissus impliqués dans la FD, et l'observation de leur relâchement après ablation, a été utilisée [8]. L'application de cette technique simple sur des embryons sauvages a permis d'aboutir à plusieurs conclusions.

Tout d'abord, et de manière attendue, l'amnioséreuse, la marge et l'ectoderme latéral sont tous trois le siège de tensions. La rupture de l'amnioséreuse entraîne un fort relâchement de l'ectoderme, révélant une activité contractile contribuant positivement à la fermeture $\left(\sigma_{\text {AS }}\right)$. À cette force de contraction s'ajoute une force engendrée par la tension du câble d'actine (TK) qui participe à l'organisation et à la linéarité de la marge. À l'inverse, l'ectoderme latéral exerce une tension contraire $\left(\sigma_{\mathrm{L} \varepsilon}\right)$ en s'opposant à l'avancement de la marge. Une conclusion majeure de ce travail est de montrer que, malgré sa proéminence, le câble d'actine ne suffit pas à produire toutes les forces requises pour la FD. La notion de fermeture par un mécanisme de «lien de bourse» (purse string) [6], fortement suggérée par la présence du câble d'actine, se révèle donc simpliste et fausse. Ce travail montre en revanche l'existence d'un réseau complexe et insoupçonné de forces et de tensions, dont la résultante est la fermeture coordonnée d'un tissu.

Les auteurs d'un article publié dans la revue Science ont eu recours à la modélisation quantitative pour comprendre la nature des forces contribuant à la FD dans un embryon sauvage, mais également pour démontrer leur réorganisation dans le cas d'un mutant affectant l'adhérence cellulaire [9]. Dans leur modèle simplifié, après le déclenchement du processus de FD, la vitesse de la progression de la marge (ou vélocité, $V$ ) est proportionnelle à la somme $\sigma_{\mathrm{L \varepsilon}}+\sigma_{\mathrm{AS}}+\mathrm{T \kappa}$ des forces agissant au point de symétrie $P$ (Figures $1 B$ et $1 C$ ). La variation au cours du temps de la hauteur $\mathrm{H}$ (Figure 1C) de l'amnioséreuse révèle une vitesse constante de la marge de $12 \mathrm{~nm} / \mathrm{s}$ en moyenne. Comme cela a été précédemment décrit, l'ablation totale de l'amnioséreuse entraîne un relâchement des tissus. À la suite de ce relâchement, la marge ré-enclenche sa progression vers la médiane dorsale, mais sa vélocité est diminuée (environ $5,5 \mathrm{~nm} / \mathrm{s}$ ). L'ensemble provoque ainsi un retard important de la fermeture, d'environ $300 \%$. En revanche, lorsque les deux points d'attache des marges (canthus) sont détruits par le rayon laser, la vélocité n'est que peu affectée (elle est d'environ 10,4 nm/s) et de nouveaux sites de contacts entre les deux fronts opposés se créent en position plus médiane. Cela confirme que le processus de «fermeture éclair» à partir des coins n'est pas le seul moteur de la fermeture, et qu'il faut à la fois le perturber et éliminer l'amnioséreuse pour bloquer complètement la FD.

La mesure de la vitesse initiale de rétraction de la marge après ablation de l'amnioséreuse permet d'établir que la force par unité de longueur exercée par l'amnioséreuse sur le câble d'actine $\left(\sigma_{\mathrm{AS}}\right)$ est équivalente à $940 \mathrm{~nm} / \mathrm{s}$. Cette force est donc 80 fois (940/12) supérieure à la force nette appliquée à la marge. Par le même type d'expériences de ruptures mécaniques, les auteurs démontrent que la force liée au câble d'actine ( $\mathrm{T} \kappa)$ est équivalente à celle de l'amnioséreuse $\left(T \kappa / \sigma_{A S}=0,7 \pm 0,3\right)$, alors que la résistance de l'épiderme latéral $\left(\sigma_{\mathrm{L \varepsilon}}\right)$ représente la force majoritaire (120 à 160 fois supérieure à la force nette). Ainsi, le rapport des forces $\sigma_{\mathrm{L} \varepsilon}$ : $\sigma_{\text {AS }}:$ Tא oscille entre $160: 80: 80$ et 120 : $80: 40$.

La modélisation du processus de «fermeture éclair» observé au niveau des «canthus» a ensuite été développée empiriquement, en prenant comme base géométrique de l'ouverture dorsale deux arcs de cercles s'entrecoupant dans le même plan (Figure 1C). Malgré cette simplification, le modèle reproduit de manière saisissante les mesures biologiques de la FD. Par régression non linéaire, il est alors possible de calculer la constante $k_{z}$ du processus de «fermeture éclair» dans différentes conditions expérimentales. Comme la contribution
A

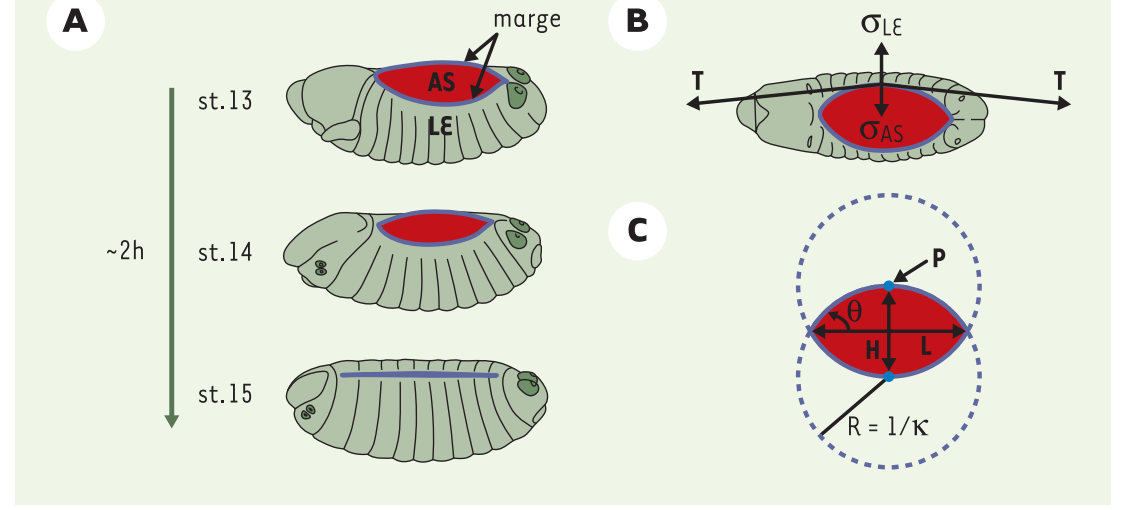

Figure 1. Modélisation de la fermeture dorsale (FD) de l'embryon de drosophile. A. Représentation schématique d'embryons en cours de fermeture, stades (st) 13 à 15. Les deux feuillets de l'épiderme latéral (en vert) délimité par une marge ( $L \varepsilon$, en bleu), migrent l'un vers l'autre en recouvrant l'amnioséreuse (AS, en rouge) et fusionnent le long de la médiane dorsale. $B$. Diagramme des forces participant à la FD. Trois forces sont en présence: forces de contraction provenant de l'amnioséreuse $\left(\sigma_{A S}\right)$ et de la tension produite dans le cable d'actine $(T)$; force de résistance de l'ectoderme latéral $\left(\sigma_{\mathrm{L} \varepsilon}\right)$. C. Base géométrique du modèle quantitatif, considérant les marges comme deux arcs de cercle s'entrecoupant. $H$ : hauteur de l'amnioséreuse; L: largeur de l'amnioséreuse; P: point de symétrie de chaque marge (en bleu); $\theta$ : angle de la marge par rapport à la médiane dorsale; R: rayon du cercle; $\kappa$ : courbure du cercle et donc de la marge. 
des «canthus» $\left(f_{z}\right)$ à la FD est directement reliée à $k_{z}$ et à la vélocité $V$, les auteurs ont pu l'évaluer à environ $33 \%$ $(1 / 3)$, alors que les forces de contraction $\left(f_{c}\right)$ engendrées par l'amnioséreuse et le câble d'actine contribuent à hauteur de $67 \%$ environ $\left(2 / 3 ; f_{c}=1-f_{z}\right)$. Lorsque l'amnioséreuse est détruite, $f_{z}$ augmente (elle est alors d'environ $50 \%$ ), alors que lorsque les deux angles de l'ouverture dorsale sont affectés, $f_{z}$ diminue fortement $(<10 \%)$. Ces résultats illustrent la validité du modèle géométrique et l'adaptabilité du système lorsqu'il est perturbé.

Les embryons mutants pour le gène myospheroid (mys) présentent un phénotype à «ciel ouvert» caractéristique d'un défaut de FD [10]. Les deux feuillets épidermiques latéraux migrent normalement, mais leur fusion n'est pas maintenue. Le gène mys code pour une sous-unité $\beta_{\text {PS }}$ intégrine, protéine transmembranaire impliquée dans l'adhérence et la communication cellulaires. La mesure et la modélisation des mutants mys montrent que la vélocité diminue d'environ $60 \%$ (environ $7,21 \mathrm{~nm} / \mathrm{s}$ ) et que les «canthus» $\left(f_{z}\right)$ ne contribuent plus qu'à hauteur de $9 \%$ à la FD, mettant à jour un rôle nouveau de mys dans le processus de «fermeture éclair». La force du modèle géométrique est de montrer que des changements compensateurs sont mis en place pour assurer la progression de la FD, à savoir une diminution relative de la résistance de l'épiderme latéral par rapport à la force exercée par l'amnioséreuse $\left(\sigma_{\mathrm{L} \varepsilon}-\sigma_{\mathrm{AS}}\right)$, ou l'augmentation de la tension du câble d'actine (T). De façon intéressante, la modélisation met donc en évidence un aspect de la fonction du gène mys demeuré invisible jusqu'alors par les approches classiques, tout en fournissant aux biologistes un jeu d'hypothèses testables expérimentalement.

Le recours à la modélisation en biologie en est encore à ses balbutiements. Comme cela est illustré dans cet exemple, l'apport des méthodes quantitatives se révèle être un outil important et un complément précieux pour l'interprétation des phénotypes observés et l'élucidation des fonctions géniques. Dans le cas de processus modèles concernant des traumatismes comme la cicatrisation, l'application de ces méthodes de biomathématiques à l'effet thérapeutique de molécules candidates peut s'avérer très prometteuse. $\diamond$ Dorsal closure, forces for morphogenesis

\section{RéFÉRENCES}

1. Martin P. Wound healingaiming for perfect skin regeneration. Science 1997; 276: 75-81.

2. Noselli S. JNK signaling and morphogenesis in Drosophila. Trends Genet 1998; 14 : 33-8.

3. Noselli S, Agnès F. Roles of the JNK signaling pathway in Drosophila morphogenesis. Curr Opin Genet Dev 1999; 9: 466-72.

4. Grose R, Martin P. Parallels between wound repair and morphogenesis in the embryo. Semin Cell Dev Biol 1999; 10: 395-404.

5. Agnès F, Noselli S. Dorsal closure in Drosophila. A genetic model for wound healing? CR Acad Sci III 1999; 322: 5-13.
6. Young PE, Richman AM, Ketchum AS, Kiehart DP. Morphogenesis in Drosophila requires nonmuscle myosin heavy chain function. Genes Dev 1993; $7: 29-41$.

7. Jacinto A, Wood W, Balayo T, Turmaine M, MartinezArias A, Martin P. Dynamic actin-based epithelial adhesion and cell matching during Drosophila dorsal closure. Curr Biol 2000; 10: 1420-6.

8. Kiehart DP, Galbraith CG, Edwards KA, Rickoll WL, Montague RA. Multiple forces contribute to cell sheet morphogenesis for dorsal closure in Drosophila. J Cell Biol 2000; 149: 471-90.

9. Hutson MS, Tokutake y, Chang MS, et al. Forces for morphogenesis investigated with laser microsurgery and quantitative modeling. Science 2003; 6 : 6 .

10. Mackrell AJ, Blumberg B, Haynes SR, Fessler JH. The lethal myospheroid gene of Drosophila encodes a membrane protein homologous to vertebrate integrin beta subunits. Proc Natl Acad Sci USA 1988; 85: 2633-7. 